\title{
Characterization of the locomotor activities of zebrafish larvae under the influence of various neuroactive drugs
}

\author{
Fei $\mathrm{Li}^{1}$, Jia Lin ${ }^{1}$, Xiuyun Liu ${ }^{1}$, Wenhui $\mathrm{Li}^{2}$, Yifeng Ding ${ }^{2}$, Yunjian Zhang ${ }^{2}$, Shuizhen Zhou ${ }^{2}$, Ning Guo ${ }^{3}$, \\ Qiang $\mathbf{L i}^{1}$
}

${ }^{1}$ Translational Medical Center for Development and Disease, Institute of Pediatrics, Shanghai Key Laboratory of Birth Defect, ${ }^{2}$ Department of Neurology, Children's Hospital of Fudan University, Shanghai 201102, China; ${ }^{3}$ Center for Chinese Medical Therapy and Systems Biology, Shanghai University of Traditional Chinese Medicine, Shanghai 201203, China

Contributions: (I) Conception and design: F Li, J Lin; (II) Administrative support: Q Li; (III) Provision of study materials or patients: Q Li; (IV) Collection and assembly of data: J Lin, X Liu; (V) Data analysis and interpretation: All authors; (VI) Manuscript writing: All authors; (VII) Final approval of manuscript: All authors.

Correspondence to: Qiang Li. Translational Medical Center for Development and Disease, Shanghai Key Laboratory of Birth Defect, Institute of Pediatrics, Children's Hospital of Fudan University, 399 Wanyuan Road, Shanghai 201102, China. Email: liq@fudan.edu.cn.

Background: Behavioral changes in animals reflect functional changes in their central nervous system. Neuroactive drugs that act on different neural pathways can induce specific behavioral responses; therefore, it is possible to infer the activities of neuroactive drugs by studying the behavioral changes induced by drugs of interest in animals.

Methods: In this study, AB strain zebrafish larvae at 7 days post fertilization (dpf) were treated with different concentrations of drugs that act on different neural pathways. Changes in the swimming distances of zebrafish larvae under different illumination conditions and the differences in locomotor activities between light and dark conditions (lighting motor index) were analyzed.

Results: Among the drugs studied, different concentrations of sulpiride had no effect on larval locomotor activity either under light or dark conditions. Progressively decreased spontaneous movements were observed in zebrafish larvae treated with increasing doses of MK-801 and valproic acid. With increasing concentrations of pentylenetetrazole and yohimbine, the spontaneous movement of larval zebrafish presented a bell-shaped response. When the illumination changed from light to dark, zebrafish larvae not treated with drugs demonstrated increased locomotor activities. However, high levels of yohimbine, pentylenetetrazole decreased the degree of change in the lighting motor index.

Conclusions: In conclusion, drugs that affect different neural pathways exert different influences on the locomotor activities of zebrafish larvae. This study presents an initial effort to establish a framework that correlates the drug activities and the behavioral responses of zebrafish larvae under drug treatments, which may provide a potential identification of the pathways of novel drugs with neurological activities through their behavioral influences.

Keywords: Zebrafish larvae; neuroactive drugs; locomotion; pathway

Submitted Mar 15, 2018. Accepted for publication Apr 09, 2018.

doi: $10.21037 /$ atm.2018.04.25

View this article at: http://dx.doi.org/10.21037/atm.2018.04.25 


\section{Introduction}

The zebrafish (Danio rerio) is a popular model organism for behavioral genetics and developmental studies, especially forward genetics (1). Because the nervous system of the zebrafish exhibits developmentally, structurally, functionally and pharmacologically conserved similarities with the mammalian nervous system, zebrafish are also widely used as experimental animals in neural and neuropharmacological studies $(2,3)$. As early as $4-5$ days post-fertilization (dpf), zebrafish larvae develop different behaviors, including emotions (anxiety and fear), learning and memory, goaldirected behavior, optokinetic responses, and prepulse inhibition (4-6).

Neuroactive drugs that interact with different receptors of the central nervous system result in diverse behavioral manifestations in zebrafish. It has been shown that acute exposure to dopamine receptor agonists induced increases in the locomotor activities of $6 \mathrm{dpf}$ zebrafish larvae, whereas dopamine receptor antagonists demonstrated opposite effects (7). This phenomenon is consistent with the findings in mammals $(8,9)$. In humans and other animals, $\gamma$-aminobutyrate (GABA) receptor antagonists have disinhibitory effects on the central nervous system, which can cause clonic or tonic-clonic seizures $(10,11)$. Low concentrations of alcohol, $\mathrm{d}$-amphetamine and cocaine were found to increase the spontaneous movement of $6 \mathrm{dpf}$ wildtype zebrafish larvae, whereas high concentrations of these drugs inhibited their locomotor activities (12).

For zebrafish, changes in illumination conditions usually result in changes in the levels of locomotor activities. It has been shown that the locomotor activities of larval zebrafish were significantly enhanced when the illumination changed from light to dark (12), and some neuroactive drugs were able to modify or even reverse this phenomenon (7). A visual motor response (VMR) assay that focused on the instantaneous changes in the swimming activities of zebrafish in response to changes in lighting conditions was previously described $(13,14)$. To characterize drug effects on locomotor activities, instead of focusing on the transient instantaneous locomotor changes caused by changes in the lighting conditions, we aimed to examine the more sustained changes in the swimming activities that are caused by differences in lighting, i.e., light and dark conditions. However, studies thus far have primarily focused on the qualitative aspect of this phenomenon; therefore, a new parameter-the lighting motor index-was devised in this work to focus on the quantitative aspect of this phenomenon.

In this study, we examined the influences of different concentrations of various neuroactive drugs on the locomotor activities and the lighting motor indexes of the zebrafish larvae under light and dark conditions. With this comparative study, we attempted to speculate a correlation between the drug activities and their behavioral influences to promote the identification of the activities of novel drugs through their effects on zebrafish locomotion.

\section{Methods}

\section{Drugs}

The drugs included MK-801; pentylenetetrazole; valproic acid sodium salt; yohimbine hydrochloride; 5,5-diphenylhydantoin sodium salt and sulpiride, which were bought from Sigma-Aldrich. Drugs were dissolved in sterile water to prepare the indicated stock solutions. Before experiment, stock solutions were diluted to appropriate concentrations.

\section{Zebrafish busbandry}

$\mathrm{AB}$ strain wild-type zebrafish were acquired from Zebrafish Facility of Children's Hospital of Fudan University, and were maintained at $28.5^{\circ} \mathrm{C}$ according to standard protocols (15). The fish were kept on a 14-h light: 10-h dark cycle (lights on at 8:00 AM; lights off at 10:00 PM). Eggs were obtained by natural spawning and were raised in groups of 50 in an incubator at $28.5^{\circ} \mathrm{C}$ from birth to $7 \mathrm{dpf}$, which was staged according to a previously published method (16). Eggs and larvae were kept under the same lighting schedule as adult zebrafish. All animal experimental procedures complied with local and international regulations. All protocols were approved by the institutional animal care committee.

\section{Bebavior tests}

Twenty-four-well plates were used to carry out the behavior tests in order to provide adequate swimming space for the zebrafish larvae. We use ZebraBox (ViewPoint Life Sciences) to record videos of zebrafish larvae activities. The experiments consisted of $60 \mathrm{~min}$ of continuous illumination (light period) followed by 5 min of darkness (dark period). The activities of zebrafish larvae during the last $10 \mathrm{~min}$ of the light period and the 5 min dark period were analyzed. The quantification of zebrafish larvae locomotion activities 

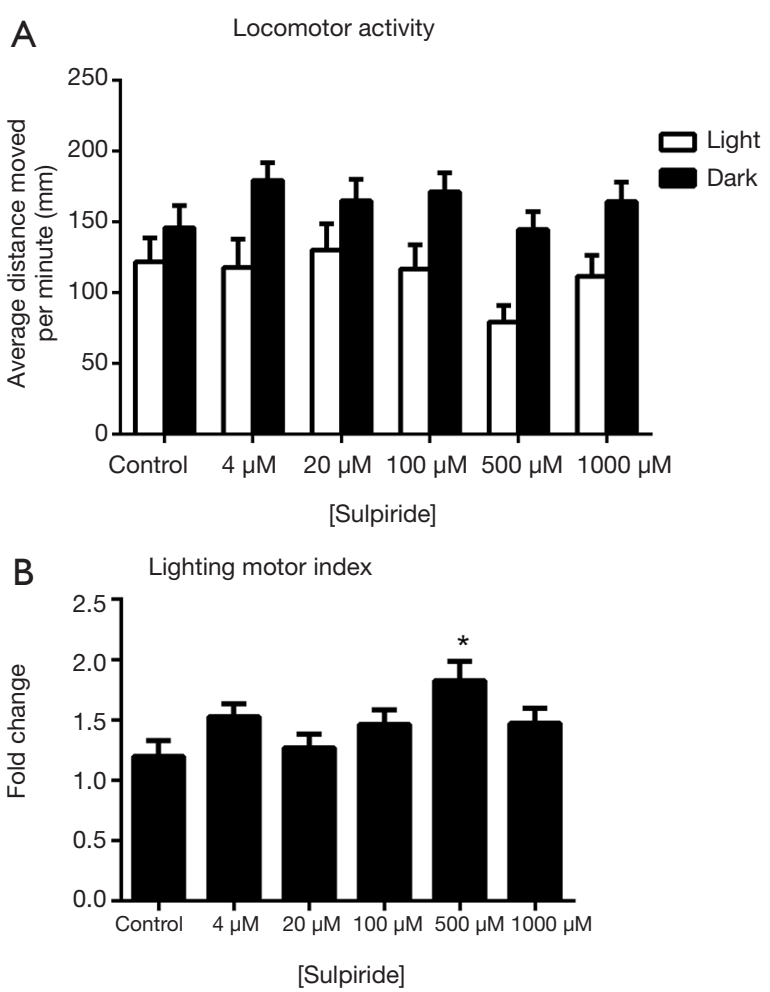

Figure 1 Effects of sulpiride on zebrafish locomotor activities (A) and the lighting motor index (B). (A) Average distances moved by zebrafish larvae within a 1 -min time bin are plotted. The horizontal axis denotes the sulpiride treatment concentration that the larvae received. The vertical axis denotes the distance moved by the zebrafish larvae in millimeters; (B) lighting motor index. The horizontal axis denotes the sulpiride treatment concentration that the larvae received. The vertical axis denotes the fold changes in the zebrafish locomotor activities under different illumination conditions. The data are presented as the mean \pm SEM; $n=32$ animals per group. *, $\mathrm{P}<0.05$, significantly differs from the control group. SEM, standard error of the mean.

was achieved using the tracking mode of ZebraLab software with recorded videos.

In this study, the swimming distances of zebrafish larvae were employed as indicators of their locomotor activities. To evaluate the influence of the illumination change from light to dark on the locomotor activity, a lighting motor index was devised as indicated below.

lighting motor index $=($ the swimming distances of the zebrafish larvae under dark conditions)/(the swimming distances of the zebrafish larvae under light conditions)

\section{Data collection and statistical analyses}

All data are presented as the mean \pm standard error of the mean (SEM). Statistical analyses were performed using GraphPad Prism software. The data for each drug were first assessed using repeated-measure two-way ANOVA, and a $\mathrm{P}$ value less than 0.05 was considered statistically significant $(\mathrm{P}<0.05)$.

\section{Results}

For sulpiride-treated zebrafish larvae, two-way ANOVA indicated that the change in illumination exerted a significant influence on their locomotor activities $\{\mathrm{F}$ $[1,372]=30.18, \mathrm{P}<0.0001\}$ but that the change in drug concentrations did not significantly affect their locomotion $\{\mathrm{F}[5,372]=1.574, \mathrm{P}=0.1666\}$ (Figure 1). The locomotor activities of the zebrafish larvae had no obviously change no matter in the light and dark conditions (Figure 1A). When the lighting motor index was considered, the zebrafish larvae treated with $500 \mu \mathrm{M}$ sulpiride demonstrated a statistically significant increase in the lighting motor index compare with the control group (Figure 1B).

For diphenylhydantoin-treated zebrafish larvae, twoway ANOVA revealed that the change in illumination significantly affected their locomotor activities $\{\mathrm{F}[1$, 372] $=37.40, \mathrm{P}<0.0001\}$. Drug treatments at different concentrations also significantly influenced their locomotor activities $\{\mathrm{F}[5,372]=4.854, \mathrm{P}=0.0003\}$ (Figure 2). Although multiple comparison tests did not identify significant influences of diphenylhydantoin treatment at different concentrations under light conditions on the locomotor activity of the zebrafish larvae, high concentrations $(500 \mu \mathrm{M})$ of diphenylhydantoin significantly reduced the locomotor activities of the larvae under dark conditions. No difference in the lighting motor index was observed between the control group and the drug-treated groups (Figure 2B).

For yohimbine-treated zebrafish larvae, two-way ANOVA analysis indicated that the change in illumination had significant influences on their locomotor activities $\{\mathrm{F}[1,372]=109.7, \mathrm{P}<0.0001\}$. In addition, drug treatment at different concentrations exerted significant influences on the locomotor activities of these zebrafish larvae $\{\mathrm{F}[5$, 372] $=217.9, \mathrm{P}<0.0001\}$ (Figure 3). Under light conditions, significantly reduced locomotor activities were observed in groups treated with $25,50,100$, or $200 \mathrm{mg} / \mathrm{L}$ yohimbine compared with the control group. Under dark conditions, $10 \mathrm{mg} / \mathrm{L}$ yohimbine treatment resulted in a significant 

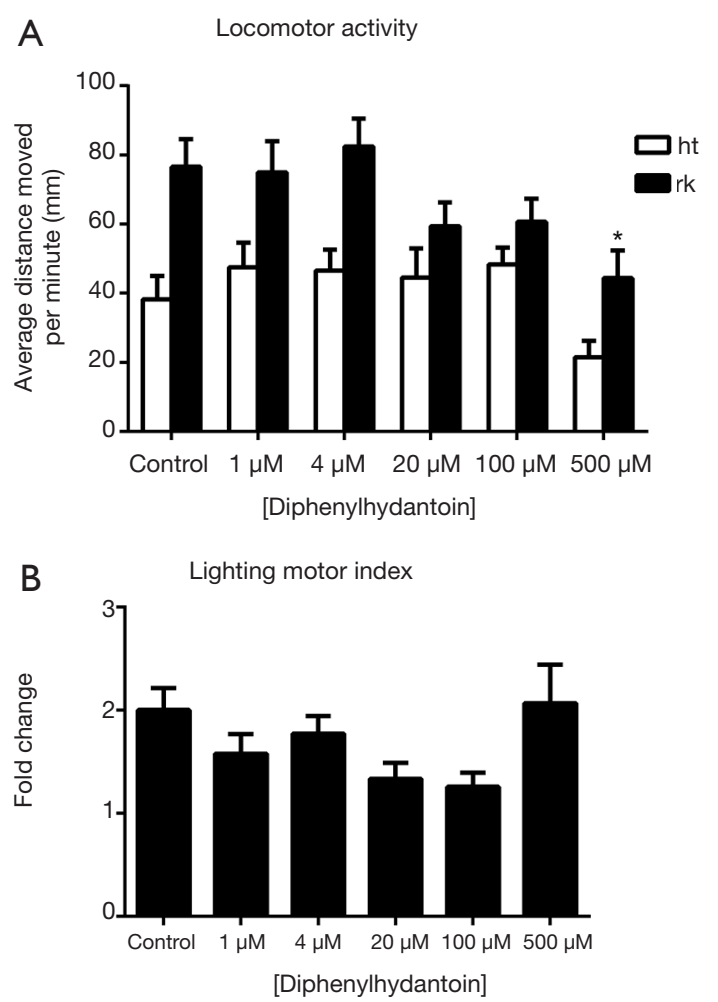

Figure 2 Effects of diphenylhydantoin on zebrafish locomotor activities (A) and the lighting motor index (B). (A) Average distances moved by the zebrafish larvae within a 1-min time bin are plotted. The horizontal axis denotes the diphenylhydantoin treatment concentration that the larvae received. The vertical axis denotes the distance moved by the zebrafish larvae in millimeters; (B) lighting motor index. The horizontal axis denotes the diphenylhydantoin treatment concentration that the larvae received. The vertical axis denotes the fold changes in the zebrafish locomotor activities under different illumination conditions. The data are presented as the mean \pm SEM; $n=32$ animals per group. *, $\mathrm{P}<0.05$, significantly differs from the control group. SEM, standard error of the mean.

increase in the locomotor activities of the zebrafish larvae, whereas further increases in the yohimbine concentration significantly inhibited the locomotor activities of the zebrafish larvae compared with the control group. When the illumination condition changed from light to dark, the zebrafish larvae treated with $25,50,100$, or $200 \mathrm{mg} / \mathrm{L}$ yohimbine presented significantly reduced lighting motor indexes (Figure 3B).

For MK-801-treated zebrafish larvae, two-way ANOVA analysis revealed significant influences of illumination conditions on their locomotor activities $\{\mathrm{F}[1,372]=35.88$,
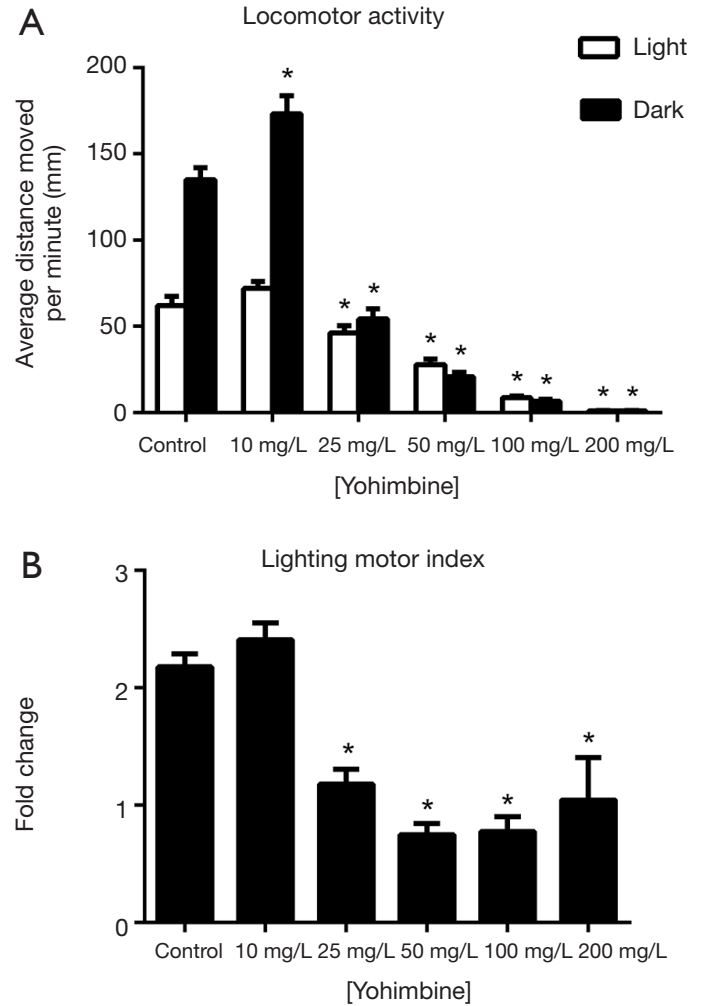

Figure 3 Effects of yohimbine on zebrafish locomotor activities (A) and the lighting motor index (B). (A) Average distances moved by the zebrafish larvae within a 1-min time bin are plotted. The horizontal axis denotes the yohimbine treatment concentration that the larvae received. The vertical axis denotes the distance moved by the zebrafish larvae in millimeters; (B) lighting motor index. The horizontal axis denotes the yohimbine treatment concentration that the larvae received. The vertical axis denotes the fold changes in the zebrafish locomotor activities under different illumination conditions. The data are presented as the mean \pm SEM; $n=32$ animals per group. ${ }^{*}, \mathrm{P}<0.05$, significantly differs from the control group. SEM, standard error of the mean.

$\mathrm{P}<0.0001\}$. The changes in drug concentrations also had significant impacts $\{\mathrm{F}[5,372]=19.45, \mathrm{P}<0.0001\}$ (Figure 4). With increasing drug concentrations, the average distance moved by the zebrafish larvae per minute demonstrated a progressive decrease compared with the control group under light conditions. Under dark conditions, increasing drug concentrations also caused a downward trend in the locomotor activities of the zebrafish larvae. The lighting motor indexes of most drug-treated groups were increased compared with that of the control group; however, no statistical significance was found (Figure 4B). 

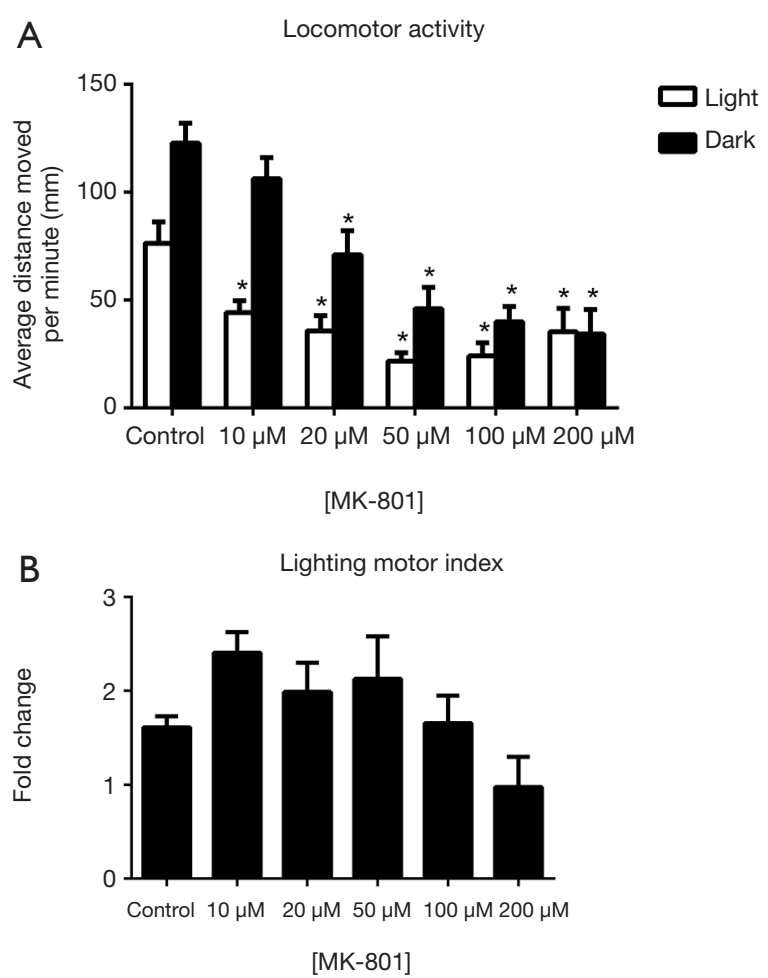

Figure 4 Effects of MK-801 on zebrafish locomotor activities (A) and the lighting motor index (B). (A) Average distances moved by the zebrafish larvae within a $1-$ min time bin are plotted. The horizontal axis denotes the MK-801 treatment concentration that the larvae received. The vertical axis denotes the distance moved by the zebrafish larvae in millimeters; (B) lighting motor index. The horizontal axis denotes the MK-801 treatment concentration that the larvae received. The vertical axis denotes the fold changes in the zebrafish locomotor activities under different illumination conditions. The data are presented as the mean \pm SEM; $n=32$ animals per group. * $\mathrm{P}<0.05$, significantly differs from the control group. SEM, standard error of the mean.

For valproic acid-treated zebrafish larvae, two-way ANOVA analysis indicated that the change in illumination conditions exerted significant effects on their locomotor activity $\{\mathrm{F}[1,372]=42.43, \mathrm{P}<0.0001\}$. The differences in drug concentrations also had significant influences on the locomotor activities of these zebrafish larvae $\{\mathrm{F}[5,372]$ $=12.71, \mathrm{P}<0.0001\}$ (Figure 5). Under both light and dark conditions, the average distance moved by the zebrafish larvae per minute was significantly reduced compared with the control groups when treated with $500 \mu \mathrm{M}$ valproic acid. However, the lighting motor indexes did not differ between the control group and the drug-treated groups (Figure 5B).
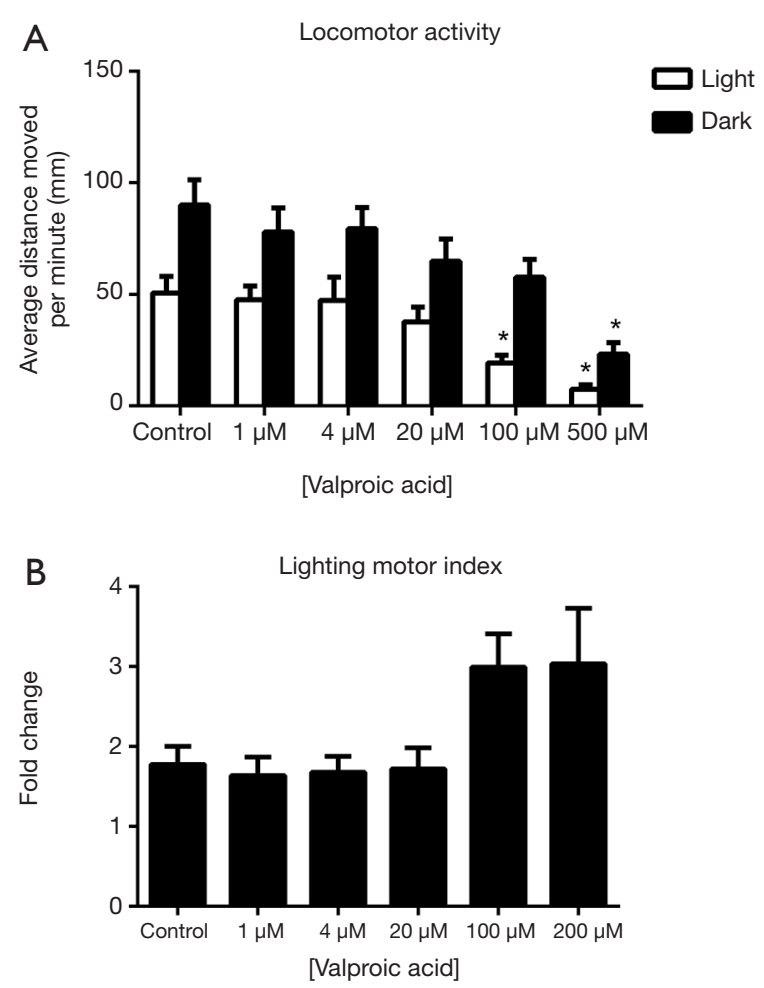

Figure 5 Effects of valproic acid on zebrafish locomotor activities (A) and the lighting motor index (B). (A) Average distances moved by the zebrafish larvae within a 1-min time bin are plotted. The horizontal axis denotes the valproic acid treatment concentration that the larvae received. The vertical axis denotes the distance moved by the zebrafish larvae in millimeters; (B) lighting motor index. The horizontal axis denotes the valproic acid treatment concentration that the larvae received. The vertical axis denotes the fold changes in the zebrafish locomotor activities under different illumination conditions. The data are presented as the mean \pm SEM; $\mathrm{n}=32$ animals per group. * $\mathrm{P}<0.05$, significantly differs from the control group. SEM, standard error of the mean.

For pentylenetetrazole-treated zebrafish larvae, two-way ANOVA analysis revealed that the change in illumination conditions had no effect on their locomotor activities $\{\mathrm{F}[1,372]=0.08475, \mathrm{P}=0.7711\}$, but the change in drug concentrations had significant effects on zebrafish locomotion $\{\mathrm{F}[5,372]=16.61, \mathrm{P}<0.0001\}$ (Figure 6). Under light conditions, a progressive increase in locomotor activities was observed in the groups treated with 1, 2, 4, or $8 \mathrm{mM}$ pentylenetetrazole, although only the locomotor activities of the 4 and $8 \mathrm{mM}$ pentylenetetrazole-treated groups significantly differed from those of the control group. When the concentration reached $16 \mathrm{mM}$, the 
Page 6 of 9

Li et al. Locomotion of zebrafish larvae under various neuroactive drugs
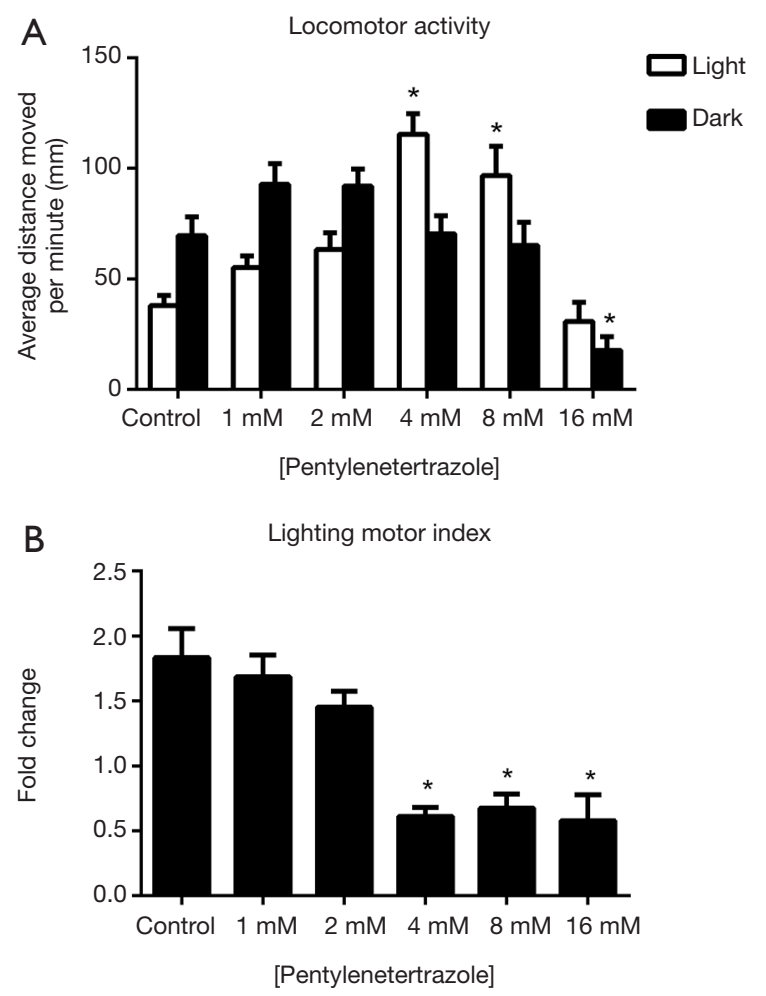

Figure 6 Effects of pentylenetetrazole on zebrafish locomotor activities (A) and the lighting motor index (B). (A) Average distances moved by the zebrafish larvae within a 1-min time bin are plotted. The horizontal axis denotes the pentylenetetrazole treatment concentration that the larvae received. The vertical axis denotes the distance moved by the zebrafish larvae in millimeters; (B) lighting motor index. The horizontal axis denotes the pentylenetetrazole treatment concentration that the larvae received. The vertical axis denotes the fold changes in the zebrafish locomotor activities under different illumination conditions. The data are presented as the mean \pm SEM; $\mathrm{n}=32$ animals per group. *, $\mathrm{P}<0.05$, significantly differs from the control group. SEM, standard error of the mean.

locomotor activities of the zebrafish larvae dropped to a level that was comparable to that of the control group. Under dark conditions, only the $16 \mathrm{mM}$ pentylenetetrazoletreated zebrafish larvae demonstrated significantly reduced locomotor activities, whereas all other drug-treated groups exhibited similar levels of locomotor activities compared to the control group. However, zebrafish larvae in the drug-treated groups $(4,8,16 \mathrm{mM})$ presented significantly decreased lighting motor indexes compared with the control group (Figure 6B).

\section{Discussion}

Not only did the larval zebrafish react differently to different drugs, but also their responses were affected by the drug concentration. At the same time, illumination also had an influence. Therefore, Table 1 was constructed to illustrate the relationships among the drugs, the targeted pathways, and the behavioral manifestations of zebrafish under different drug treatments. The aim of this study was to present an initial effort to establish a framework that correlates the drug activities and the behavioral responses of zebrafish larvae under drug treatments, which may provide a potential identification of the pathways of novel drugs with neurological activities through their behavioral influences.

With increasing concentrations of yohimbine, the spontaneous movement of zebrafish larvae was markedly decreased, regardless of illumination. These observations were similar to other studies with rodents. When Male hooded Lister rats were acutely treated with yohimbine at 2.5 and $5 \mathrm{mg} / \mathrm{kg}$, the rats showed decreased locomotor activities (17). Male Sprague-Dawley rats with yohimbine treatment below $2 \mathrm{mg} / \mathrm{kg}$ demonstrated increased locomotor activities, whereas yohimbine treatment at higher doses resulted in decreased locomotion (18). One possible explanation is that yohimbine inhibits the activity of $\alpha 2$-adrenaline receptors, which leads to inhibition of these neurons. Sulpiride, a specific D2 dopamine receptor antagonist, is another drug tested in the current study, and we found that sulpiride did not affect zebrafish larval locomotor activities under the current protocol, irrespective of whether the light or dark condition was imposed. Few studies have examined the influence of sulpiride on the locomotor activities of model organisms. However, a study examining the effects of haloperidol, a drug that functions on the same neurological pathway as sulpiride, found no effect on the locomotor activity of zebrafish larvae in the first 80 minutes when haloperidol was applied at different concentrations. This finding was consistent with our observation. However, after 80 minutes, different concentrations of haloperidol had different effects on the spontaneous movement of zebrafish larvae (7), with high concentrations exerting an inhibitory effect on locomotor activity (7). As our purpose was to establish an effective platform of drug screening, a longer protocol was not the optimal choice. However, it would be advisable to include a second round of screening with longer protocols for drugs that did not show any observable effects during the first round of rapid screening. 
Table 1 Summary of drug effects on the locomotor activity, including the activity and the possible target

\begin{tabular}{lllll}
\hline Compound name & Activities & Influence under light & Influence under dark ${ }^{\star \star}$ & Target $^{*}$ \\
\hline Sulpiride & Dopamine receptor antagonist & No difference & No difference & Excitatory neuron \\
5,5-Diphenylhydantoin & Sodium channel antagonist & No difference & Downward trend & Excitatory neuron \\
MK-801 & NMDA receptor antagonist & Downward trend & Downward trend & Excitatory neuron \\
Valproic acid & Histone acetylation enzyme inhibitor & Downward trend & Downward trend & - \\
Yohimbine & $\alpha_{2}$-adrenaline receptor antagonist & Downward trend & Downward trend & - \\
Pentylenetetrazole & $\gamma$-aminobutyric acid receptor antagonist & Upward trend & Upward trend & Inhibitory neuron \\
\hline
\end{tabular}

*, The influence of locomotion under the light condition; ${ }^{*}$, the influence of locomotion under the dark condition; -, there is no clear definition of the types of neurons.

GABA is an inhibitory neurotransmitter of the central nervous system, and pentylenetetrazole, a GABA receptor antagonist, has been shown in our previous work to increase the locomotion of larval zebrafish under continuous illumination (19). In addition, progressively increased bodily movements were observed in adult zebrafish treated with increasing concentrations of pentylenetetrazole $(5,7.5,10$ and $15 \mathrm{mM}$ ) (11), and seizure-like behaviors were observed in zebrafish treated with $15 \mathrm{mM}$ pentylenetetrazole (11). These observations share great similarities with our current results. In this present study, progressively increased locomotor activities were observed in zebrafish larvae treated with increasing concentrations of pentylenetetrazole, regardless of illumination, though an obvious but nonsignificant decrease in the spontaneous movement of the larvae was observed at $16 \mathrm{mM}$ pentylenetetrazole. This could possibly be due to the toxicity to the nervous system of the drug at high concentrations $(7,19)$. The alterations in startle response patterns immediately following lightdark transitions reflects changes in stress/anxiety levels of animals $(20,21)$. Pentylenetetrazole-treated zebrafish larvae exhibited significantly decreased anxiety levels during the light-dark transition, as indicated by decreases in the lighting motor index. This is in accordance with a previous study (20), in which $5 \mathrm{dpf}$ zebrafish larvae were treated with $5 \mathrm{mM}$ pentylenetetrazole. Picrotoxin is another drug that acts on the same neural pathway as pentylenetetrazole. In a different work by our group, $5 \mathrm{dpf}$ zebrafish larvae under picrotoxin treatment showed progressively enhanced locomotor activities with increasing picrotoxin concentrations under the light condition; in contrast, an inhibitory effect of picrotoxin treatment on locomotor activities was observed under the highest picrotoxin concentration tested under the light condition. However, no effect on spontaneous movement by zebrafish larvae under the dark condition was observed (22). Male albino rats that were acutely treated with $1.5 \mathrm{mg} / \mathrm{kg}$ picrotoxin intraperitoneally demonstrated epileptic seizures after 2030 minutes (23). Hyper-synchronous (HYP) activities were observed in electroencephalography recordings in male Sprague-Dawley rats receiving intraperitoneal injections of picrotoxin at $3-5 \mathrm{mg} / \mathrm{kg}$ (24). These observations are the result of a dis-inhibited nervous system caused by GABA receptor antagonists.

Discrepancies between our results and previously publish works were also noted. In the current study, decreased locomotor activities were observed in $7 \mathrm{dpf}$ larvae with acute MK-801 treatment (20, 50, 100 and $200 \mu \mathrm{M})$ under both light and dark conditions. Mice treated with $0.05 \mathrm{mg} / \mathrm{kg}$ MK-801 from 7 to 49 days postpartum demonstrated decreased locomotor activities (25). However, in other studies, acute MK-801 treatments resulted in increased locomotor activities in both zebrafish and rodents. Increases in locomotor activities were observed in Male C57BL/6 mice with 0.3 and $0.6 \mathrm{mg} / \mathrm{kg}$ MK-801 treatment (26), and 5 dpf zebrafish larvae demonstrated increased swimming activities when treated with $50 \mu \mathrm{M}$ MK-801 (27). Increases in locomotor activities were also observed in adult zebrafish with $20 \mu \mathrm{M}$ MK-801 treatment (28). These differences between our results and the results of others indicate the influences of species, genetic background, and age on the neurological effects of various drugs.

In our study, valproic acid and 5,5-diphenylhydantoin were the two drugs showing acute influences on spontaneous locomotor activities but not well documented in previously published works. It is well known that prenatal exposure to valproic acid could result in neurodevelopmental disorder during adolescence (29). The offspring of pregnant Wistar 
rats that received an intraperitoneal injection of $600 \mathrm{mg} / \mathrm{kg}$ valproic acid demonstrated declined spontaneous movements during puberty and maturity compared with rat offspring not treated with valproic acid (30). In our work, high concentration $(500 \mu \mathrm{M})$ of valproic acid significantly reduced the spontaneous movement of zebrafish larvae under both light and dark conditions. This observation is in agreement with the role of valproic acid as an anti-epileptic drug. Our study found that $500 \mu M$ 5,5-diphenylhydantoin decreased the spontaneous movement of the zebrafish larvae under both light and dark conditions. A previous study demonstrated that 5 and $10 \mathrm{mg} / \mathrm{kg}$ diphenylhydantoin could inhibit methylphenidate-induced hyper-locomotor activities in male Swiss albino mice (31). Thus far, few studies have examined the locomotor influences of diphenylhydantoin on model organisms. Therefore, our work provides a foundation for further studies on the activities of these two drugs.

\section{Conclusions}

Various zebrafish behavioral paradigms have been devised to explore the influences of neuroactive drugs on the behavioral manifestations of a spectrum of neurological processes. Our purpose was to establish a correlation between drug actions and their behavioral influences using a high-throughput screening protocol to facilitate as well as to expedite the identification of the actions of novel drugs through their behavioral manifestations. Therefore, zebrafish larvae at $7 \mathrm{dpf}$, an acute drug treatment regimen, a short 65 min experimental duration, simple behavioral tests, and an automated data acquisition system were used to maximize the turnover of the experiments. This study presents an initial effort to establish a framework that correlates the drug activities and the behavioral responses of zebrafish larvae under drug treatments, which could facilitate the identification of the mechanisms of novel drugs with neurological activities through their behavioral influences.

\section{Acknowledgements}

Funding: This work was supported by grants from the National Natural Science Foundation of China [81771632] and the National Key Research and Development Program [2016YFC1000500] to Q Li; and by Shanghai Key Laboratory of Birth Defect [13DZ2260600] to N Guo. This work was also supported by grants to $\mathrm{X}$ Liu from the
National Natural Science Foundation of China [81601329].

\section{Footnote}

Conflicts of Interest: The authors have no conflicts of interest to declare.

\section{References}

1. Sison M, Cawker J, Buske C, et al. Fishing for genes influencing vertebrate behavior: zebrafish making headway. Lab Anim (NY) 2006;35:33-9.

2. Anderson KV, Ingham PW. The transformation of the model organism: a decade of developmental genetics. Nat Genet 2003;33 Suppl:285-93.

3. Xi Y, Ryan J, Noble S, et al. Impaired dopaminergic neuron development and locomotor function in zebrafish with loss of pink1 function. Eur J Neurosci 2010;31:623-33.

4. Brockerhoff SE, Hurley JB, Janssen-Bienhold U, et al. A behavioral screen for isolating zebrafish mutants with visual system defects. Proc Natl Acad Sci U S A 1995;92:10545-9.

5. Burgess HA, Granato M. Sensorimotor gating in larval zebrafish. J Neurosci 2007;27:4984-94.

6. Guo S. Linking genes to brain, behavior and neurological diseases: what can we learn from zebrafish? Genes Brain Behav 2004;3:63-74.

7. Irons TD, Kelly PE, Hunter DL, et al. Acute administration of dopaminergic drugs has differential effects on locomotion in larval zebrafish. Pharmacol Biochem Behav 2013;103:792-813.

8. Beninger RJ, Mazurski EJ, Hoffman DC. Receptor subtype-specific dopaminergic agents and unconditioned behavior. Pol J Pharmacol Pharm 1991;43:507-28.

9. Sobrian SK, Jones BL, Varghese S, et al. Behavioral response profiles following drug challenge with dopamine receptor subtype agonists and antagonists in developing rat. Neurotoxicol Teratol 2003;25:311-28.

10. Baraban SC, Taylor MR, Castro PA, et al. Pentylenetetrazole induced changes in zebrafish behavior, neural activity and c-fos expression. Neuroscience 2005;131:759-68.

11. Mussulini BH, Leite CE, Zenki KC, et al. Seizures induced by pentylenetetrazole in the adult zebrafish: a detailed behavioral characterization. PLoS One 2013;8:e54515.

12. Irons TD, MacPhail RC, Hunter DL, et al. Acute neuroactive drug exposures alter locomotor activity in 
larval zebrafish. Neurotoxicol Teratol 2010;32:84-90.

13. Deeti S, O'Farrell S, Kennedy BN. Early safety assessment of human oculotoxic drugs using the zebrafish visualmotor response. J Pharmacol Toxicol Methods 2014;69:1-8.

14. Emran F, Rihel J, Dowling JE. A behavioral assay to measure responsiveness of zebrafish to changes in light intensities. J Vis Exp 2008;(20).

15. Westerfield M. The zebrafish book: a guide for the laboratory use of zebrafish (Brachydanio rerio). Eugene, OR: M. Westerfield, 1993.

16. Kimmel CB, Ballard WW, Kimmel SR, et al. Stages of embryonic development of the zebrafish. Dev Dyn 1995;203:253-310.

17. Johnston AL, Baldwin HA, File SE. Measures of anxiety and stress in the rat following chronic treatment with yohimbine. J Psychopharmacol 1988;2:33-8.

18. Zaretsky DV, Zaretskaia MV, DiMicco JA, et al. Yohimbine is a 5 -HT1A agonist in rats in doses exceeding $1 \mathrm{mg} / \mathrm{kg}$. Neurosci Lett 2015;606:215-9.

19. Peng X, Lin J, Zhu YD, et al. Anxiety-related behavioral responses of pentylenetetrazole-treated zebrafish larvae to light-dark transitions. Pharmacol Biochem Behav 2016;145:55-65.

20. Ellis LD, Seibert J, Soanes KH. Distinct models of induced hyperactivity in zebrafish larvae. Brain Res 2012;1449:46-59.

21. Pittman JT, Lott CS. Startle response memory and hippocampal changes in adult zebrafish pharmacologicallyinduced to exhibit anxiety/depression-like behaviors. Physiol Behav 2014;123:174-9.

22. Yang X, Lin J, Peng X, et al. Effects of picrotoxin on zebrafish larvae behaviors: A comparison study with PTZ. Epilepsy Behav 2017;70:224-31.

Cite this article as: Li F, Lin J, Liu X, Li W, Ding Y, Zhang Y, Zhou S, Guo N, Li Q. Characterization of the locomotor activities of zebrafish larvae under the influence of various neuroactive drugs. Ann Transl Med 2018;6(10):173. doi: 10.21037/ atm.2018.04.25
23. Acharya MM, Khamesra SH, Katyare SS. Picrotoxininduced convulsions and lysosomal function in the rat brain. Indian J Clin Biochem 2005;20:56-60.

24. Salami P, Levesque M, Gotman J, et al. Distinct EEG seizure patterns reflect different seizure generation mechanisms. J Neurophysiol 2015;113:2840-4.

25. Latysheva NV, Rayevsky KS. Chronic neonatal N-methylD-aspartate receptor blockade induces learning deficits and transient hypoactivity in young rats. Prog Neuropsychopharmacol Biol Psychiatry 2003;27:787-94.

26. Wu J, Zou H, Strong JA, et al. Bimodal effects of MK801 on locomotion and stereotypy in C57BL/6 mice. Psychopharmacology (Berl) 2005;177:256-63.

27. Chen J, Patel R, Friedman TC, et al. The Behavioral and Pharmacological Actions of NMDA Receptor Antagonism are Conserved in Zebrafish Larvae. Int J Comp Psychol 2010;23:82-90.

28. Seibt KJ, Oliveira Rda L, Zimmermann FF, et al. Antipsychotic drugs prevent the motor hyperactivity induced by psychotomimetic MK-801 in zebrafish (Danio rerio). Behav Brain Res 2010;214:417-22.

29. Rice D, Barone S Jr. Critical periods of vulnerability for the developing nervous system: evidence from humans and animal models. Environ Health Perspect 2000;108 Suppl 3:511-33.

30. Olexova L, Senko T, Stefanik P, et al. Habituation of exploratory behaviour in VPA rats: animal model of autism. Interdiscip Toxicol 2013;6:222-7.

31. Tonelli DA, Pereira M, Siba IP, et al. The antimaniclike effect of phenytoin and carbamazepine on methylphenidate-induced hyperlocomotion: role of voltage-gated sodium channels. Fundam Clin Pharmacol 2013;27:650-5. 